\title{
BMJ
}

\section{Short term and intermediate term comparison of endarterectomy versus stenting for carotid artery stenosis: systematic review and meta-analysis of randomised controlled clinical trials}

\author{
Pascal Meier, clinical and research fellow, ${ }^{1,2}$ Guido Knapp, senior scientist, ${ }^{3}$ Umesh Tamhane, research fellow, \\ Seemant Chaturvedi, professor of neurology, ${ }^{4}$ Hitinder S Gurm, assistant professor of cardiology ${ }^{1,2}$
}

\begin{abstract}
University of Michigan
Cardiovascular Center, Floor 2A

394, 1500 E Medical Center Drive,

Ann Arbor, MI 48109-5853, USA

${ }^{2}$ Veterans Affairs Ann Arbor Healthcare System, Ann Arbor, MI

${ }^{3}$ Department of Statistics, TU

Dortmund University, Dortmund, Germany

${ }^{4}$ Wayne State University/Detroit Medical Center, Department of Neurology, Detroit, MI, USA Correspondence to: H S Gurm
\end{abstract} hgurm@umich.med.edu

Cite this as: BMJ 2010;340:c467 doi:10.1136/bmi.c467

\section{ABSTRACT}

Objective To evaluate the relative short term safety and intermediate term efficacy of carotid endarterectomy versus carotid artery stenting.

Design Systematic review and meta-analysis.

Data sources BIOSIS, Embase, Medline, the Cochrane central register of controlled trials, International Pharmaceutical Abstracts database, ISI Web of Science, and Google scholar and bibliographies, from 1 January 1990 to 25 July 2009.

Study selection Randomised controlled trials comparing carotid endarterectomy with carotid artery stenting in patients with carotid artery stenosis with or without symptoms.

Data extraction Primary end point was a composite of mortality or stroke. Secondary end points were death, stroke, myocardial infarction, or facial neuropathy (as individual end points), and mortality or disabling stroke (as a composite end point).

Data synthesis 11 trials were included (4796 patients); 10 reported on short term outcomes $(n=4709)$ and nine on intermediate term outcomes (1-4 years). The periprocedural risk of mortality or stroke was lower for carotid endarterectomy (odds ratio $0.67,95 \%$ confidence interval 0.47 to $0.95 ; \mathrm{P}=0.025$ ) than for carotid stenting, mainly because of a decreased risk of stroke $(0.65,0.43$ to $1.00 ; P=0.049)$, whereas the risk of death $(1.14,0.56$ to 2.31; $\mathrm{P}=0.727)$ and the composite end point mortality or disabling stroke $(0.74,0.53$ to $1.05 ; \mathrm{P}=0.088)$ did not differ significantly. The odds of periprocedural myocardial infarction $(2.69,1.06$ to $6.79 ; \mathrm{P}=0.036)$ or cranial nerve injury $(10.2,4.0$ to 26.1 ; P<0.001) was higher in the carotid endarterectomy group than in the carotid stenting group. In the intermediate term, the two treatments did not differ significantly for stroke or death (hazard ratio $0.90,95 \%$ confidence interval 0.74 to $1.1 ; P=0.314$ ). Conclusions Carotid endarterectomy was found to be superior to carotid artery stenting for short term outcomes but the difference was not significant for intermediate term outcomes; this difference was mainly driven by nondisabling stroke. Significantly fewer cranial nerve injuries and myocardial infarctions occurred with carotid artery stenting.

\section{INTRODUCTION}

Carotid stenosis is responsible for around 20\% of strokes in the adult population. ${ }^{1}$ Treatment of carotid stenosis therefore lies in decreasing the risk of stroke or stroke related deaths. ${ }^{2}$ The main treatment is currently carotid endarterectomy, a procedure that has been shown to be superior to medical therapy in the prevention of stroke and death in patients with carotid stenosis with and without symptoms. ${ }^{3}$ Carotid angioplasty or carotid artery stenting has been emerging as a newer and less invasive alternative. Initial studies indicate that this approach is feasible, safe, and effective. However, the results of randomised controlled trials comparing carotid artery stenting with carotid endarterectomy are ambiguous. ${ }^{45} \mathrm{~A}$ recent meta-analysis of randomised controlled trials reported an increased risk of stroke or death within 30 days of carotid artery stenting compared with carotid endarterectomy. ${ }^{6}$ In addition, a newer large observational study ${ }^{7}$ and a multicentre randomised controlled trial ${ }^{8}$ found a higher periprocedural ( $<30$ days) incidence of stroke with carotid artery stenting compared with carotid endarterectomy. In this observational study, treatment allocation was not random and therefore the study is prone to confounding ${ }^{9}$; patients at high surgical risk underwent carotid artery stenting and had inherently higher risks of myocardial infarction and death.

We evaluated the short term (periprocedural) safety and intermediate term efficacy of carotid artery stenting versus carotid endarterectomy for stroke or death in patients with carotid stenosis with or without symptoms.

\section{METHODS}

The study was done according to the preferred reporting items for systematic reviews and meta-analyses (PRISMA) guidelines. ${ }^{10}$ Two authors (HSG and PM) planned and designed the study and created an electronic database with variables of interest (Microsoft 
Potentially relevant studies identified $(n=143)$ :

Search of databases $(n=129)$

Search through other sources $(n=14)$

Stage 1: Review of

title and abstract

Study abstracts screened after removing duplicates $(n=114)$

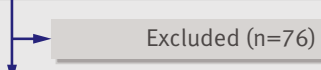

Full text articles assessed for eligibility $(n=38)$

Stage 2: Full text review or contact with study authors

Randomised controlled trials included in meta-analysis $(n=11)$

Fig 1| Outline of search and selection strategy

Excel). We defined the primary and secondary end points, variables of interest, and search strategy (databases, sources for unpublished data) in a strategy outline (details available on request).

We included randomised controlled trials that compared carotid endarterectomy with carotid artery stenting (with and without the use of cerebral protective devices) in patients with carotid stenosis with and without symptoms. The outcomes of primary interest were death and stroke at least one year after a procedure as well as myocardial infarction within 30 days of a procedure. We included randomised controlled trials regardless of their publication status, language, size, or duration of follow-up.

We searched BIOSIS, Embase, Medline, the Cochrane central register of controlled trials, International Pharmaceutical Abstracts database, ISI Web of Science, and Google scholar from 1 January 1990 to 25 July 2009. See web extra table 1 for details of the search strategy on Medline. We used similar but adapted search terms for the other databases.

We also searched abstract lists and conference proceedings of the 2006-9 scientific sessions of the
American College of Cardiology, the European Society of Cardiology, the 2006-8 proceedings of the Transcatheter Cardiovascular Therapeutics, and the American Heart Association. We also considered published review articles, editorials, and internet based sources of information (www.tctmd.com, www.the heart.org). Medical subject headings and keyword searches included the terms carotid endarterectomy, carotid artery stenting, stroke, myocardial infarction, and death. We reviewed the reference lists of selected articles for other potentially relevant citations. Authors of selected studies were contacted for further information if necessary, such as the hazard ratios for intermediate term data.

\section{Study selection}

Two investigators (HSG and PM) identified potential studies and assessed them for eligibility using a two step process. Firstly, they independently reviewed the titles and abstracts of all citations to identify potentially relevant studies and to exclude duplicates. Secondly, they reviewed the corresponding publications in full text to assess if studies met the inclusion criteria - that is, randomised controlled trials that directly compared carotid endarterectomy with carotid artery stenting; had stroke or death, or both as end points; and had at least 30 days of follow-up. Reviewers were not blinded to study authors or outcomes. The final inclusion of studies was based on agreement between the reviewers.

\section{Data extraction and quality assessment}

Three investigators (PM, UT, and HSG) used the standardised extraction database (Microsoft Excel) to extract information on outcome (numbers of patients, event rates for periprocedural and intermediate term death and stroke, both as single end points and as a composite end point) with hazard ratios; periprocedural death or disabling stroke (composite end point); myocardial infarction; and facial neuropathy as well as

\section{Characteristics of studies included in meta-analysis}

\begin{tabular}{|c|c|c|c|c|c|c|c|}
\hline \multirow[b]{2}{*}{ Study (years) } & \multirow{2}{*}{$\begin{array}{c}\text { Short term/ } \\
\text { intermediate } \\
\text { term data }\end{array}$} & \multirow[b]{2}{*}{ Interventions (No of patients) } & \multirow[b]{2}{*}{$\begin{array}{l}\text { Duration of } \\
\text { follow-up }\end{array}$} & \multicolumn{3}{|c|}{ Proportion of patients (\%) } & \multirow[b]{2}{*}{$\begin{array}{c}\text { Mean age } \\
\text { (years) }\end{array}$} \\
\hline & & & & $\begin{array}{c}\text { Distal } \\
\text { protection }\end{array}$ & $\begin{array}{c}\text { Stent } \\
\text { implanted }\end{array}$ & Asymptomatic & \\
\hline Naylor et $\mathrm{al}^{29}(1998)$ & Yes/no & Carotid endarterectomy $(n=12) v$ carotid artery stenting $(n=11)$ & 30 days & 0 & 100 & 0 & $66.7 v$ NA \\
\hline Wallstent $^{26}(2001)$ & Yes/yes & Carotid endarterectomy $(n=112) v$ carotid artery stenting $(n=107)$ & 12 months & 0 & 100 & 0 & $70 \vee 66.5$ \\
\hline CAVATAS $^{25}(2001)$ & Yes/yes & Carotid endarterectomy $(n=253) v$ carotid artery stenting $(n=251)$ & 23.4 months & 0 & 26 & 3 & $67 v 67$ \\
\hline 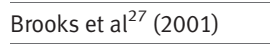 & Yes/yes & Carotid endarterectomy $(n=51) v$ carotid artery stenting $(n=53)$ & 24 months & 0 & 100 & 0 & $69.6 \vee 66.4$ \\
\hline Brooks et $\mathrm{al}^{30}(2004)$ & Yes/no & Carotid endarterectomy $(n=42) v$ carotid artery stenting $(n=43)$ & 48 months & 0 & 100 & 100 & $69.9 \vee 66.6$ \\
\hline SAPPHIRE $^{22} 33(2004 / 8)$ & Yes/yes & Carotid endarterectomy $(n=167) v$ carotid artery stenting $(n=167)$ & 36 months & 95.6 & 100 & 71.2 & $72.6 \vee 72.5$ \\
\hline EVA-3S $2132(2006 / 8)$ & Yes/yes & Carotid endarterectomy $(n=262) v$ carotid artery stenting $(n=265)$ & 42.5 months & 92 & 100 & 0 & $70.2 \vee 69.1$ \\
\hline $\mathrm{SPACE}^{23}(2007)$ & Yes/yes & Carotid endarterectomy $(n=584) v$ carotid artery stenting $(n=599)$ & 48 months & 27 & 100 & 0 & $68.2 v 67.6$ \\
\hline BACASS $^{28}(2006)$ & Yes/yes & Carotid endarterectomy $(n=10) v$ carotid artery stenting $(n=10)$ & 48 months & 100 & 100 & 0 & $71 \vee 69$ \\
\hline Steinbauer et $\mathrm{al}^{24}$ (2008) & No/yes & Carotid endarterectomy $(n=44) v$ carotid artery stenting $(n=43)$ & 65 months & 0 & 100 & 0 & $68.4 \vee 67.9$ \\
\hline $\mathrm{ICSS}^{8}(2009)$ & Yes/no & Carotid endarterectomy $(\mathrm{n}=857) v$ carotid artery stenting $(\mathrm{n}=853)$ & 30 days & 80 & 100 & 0 & NA \\
\hline
\end{tabular}

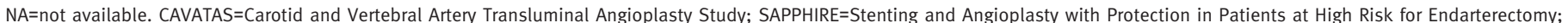
EVA-3S=Endarterectomy Versus Angioplasty in patients with Symptomatic Severe carotid Stenosis; SPACE=Stent-Supported Percutaneous Angioplasty of the Carotid Artery versus Endarterectomy; BACASS=Basel Carotid Artery Stenting Study; ICSS=International Carotid Stenting Study. 


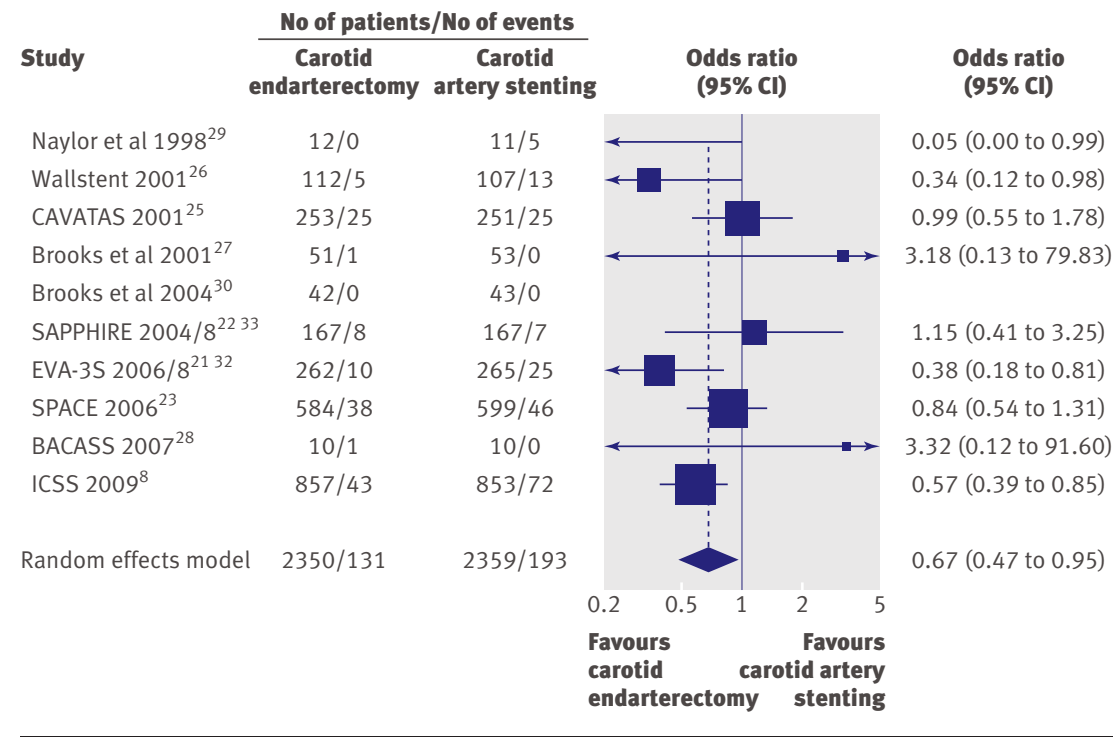

Fig 2 | Forest plot of odds ratios of risk for composite of stroke or death within 30 days of carotid endarterectomy versus carotid artery stenting. ICSS also included myocardial infarctions (three for stenting, four for endarterectomy) in this end point. See footnote to table for full title of studies for differential follow-up intervals and patients who were lost to follow-up. All analyses were done on an intention to treat basis. When an event did not occur in one group we used continuity correction. ${ }^{15} \mathrm{We}$ evaluated the presence of heterogeneity across trials with the Q and Higgins's and Thompson's I ${ }^{2}$ statistics. $\mathrm{I}^{2}$ can be interpreted as the percentage of variability due to heterogeneity between studies rather than to sampling error. To assess the effect of individual studies on the summary estimate of effect, we carried out an influence analysis, in which we recalculated the pooled estimates by omitting one study at a time. We assessed publication bias visually (funnel plot) and by formal tests (Egger's test of intercept and the arcsine test). ${ }^{16-18}$ Average weighted incidence of events is presented for both treatments; calculation was based on a random effect analysis using the inverse variance method and a Freeman-Tukey double arcsine transformation. ${ }^{19}$ All data are presented with 95\% confidence intervals. Two investigators (GK and PM) carried out analyses independently using $\mathrm{R}$, version 2.9.0, package meta and metaphor, and SAS version 9.1 (Proc nlmixed). ${ }^{1320}$

\section{RESULTS}

Of 58 full text articles reviewed, 11 trials met the inclusion criteria (fig 1). ${ }^{821-30}$ Ten reported on short term outcomes and nine on intermediate term outcomes; among those, one reported exclusively on intermediate term outcomes. ${ }^{24}$ The Carotid Revascularization using Endarterectomy or Stenting Systems (CARESS) trial was not included in this analysis as treatment assignment was not randomised. ${ }^{31}$ The table summarises the characteristics of the 11 trials.

\section{Periprocedural outcome}

The weighted average incidence of periprocedural death or stroke was 5.4\% (95\% confidence interval $4.0 \%$ to $7.0 \%$ ) for carotid endarterectomy and 7.3\% $(4.9 \%$ to $10.1 \%)$ for carotid artery stenting. The risk was significantly lower for carotid endarterectomy compared with carotid artery stenting (odds ratio $0.67,95 \%$ confidence interval 0.47 to $0.95 ; \mathrm{P}=0.025$; $\mathrm{I}^{2}=37.4 \% ; \mathrm{P}=0.119$ ) (fig 2). The studies defined periprocedural stroke differently. The Carotid and Vertebral Artery Transluminal Angioplasty Study (CAVATAS) only counted a stroke event if symptoms lasted longer then seven days. ${ }^{25}$ Other studies, such as the Basel Carotid Artery Stenting Study (BACASS), defined stroke as a neurological deterioration with persistence over 24 hours. ${ }^{28}$ Neither funnel plot, Egger's plot (see web extra fig 1), nor formal tests (Egger's and arcsine test) indicated potential publication bias or small study bias $(\mathrm{P}=0.932$ and $\mathrm{P}=0.989$, respectively).

Further analyses to evaluate heterogeneity of study results showed an important change in the comparative efficacy of the two procedures over time. The distinct inferiority of carotid artery stenting compared with carotid endarterectomy shown early on by cumulative meta-analysis diminished over time when newer trials were added sequentially (see web extra fig 2). Another factor was the high number of trials 


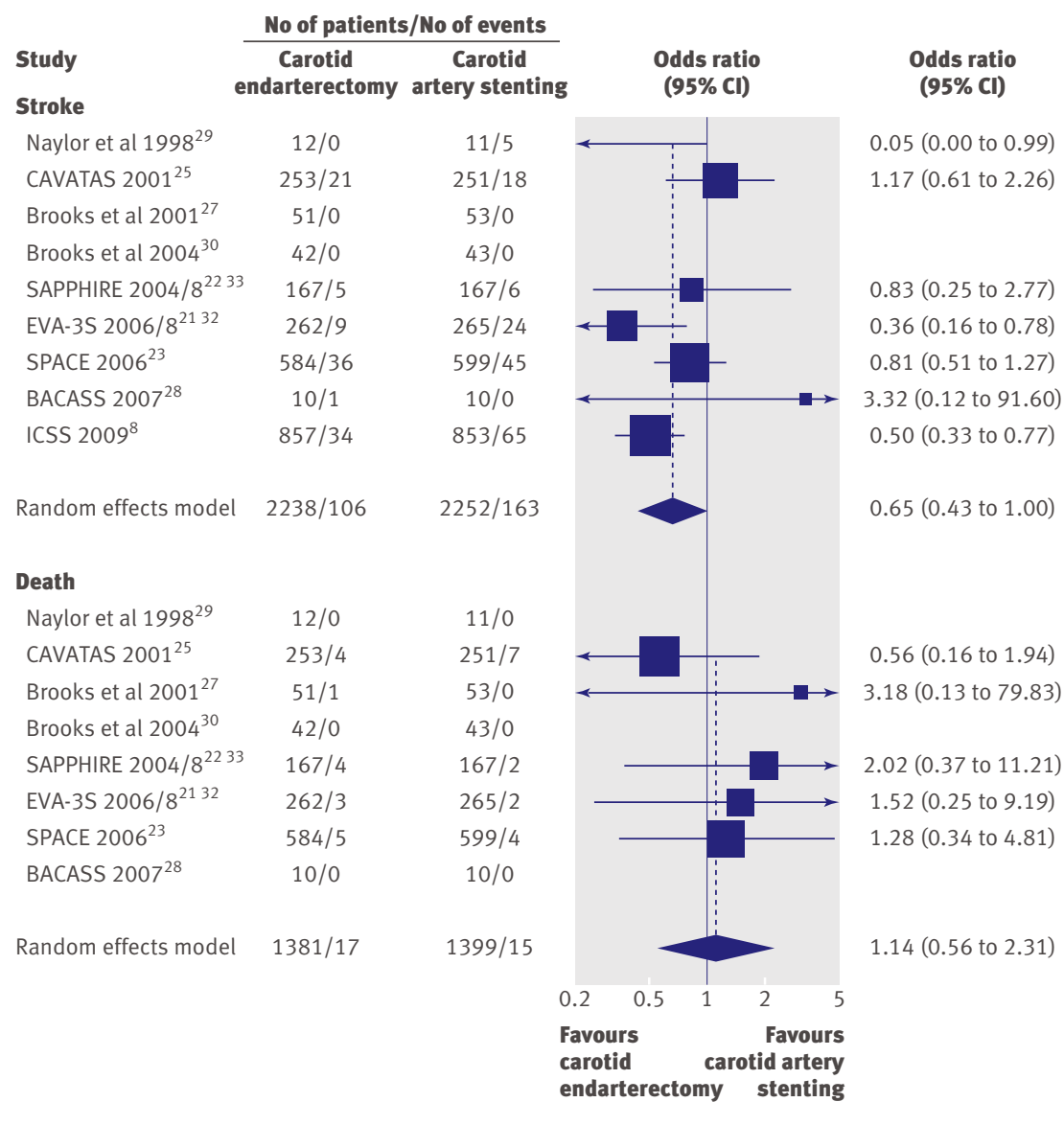

Fig 3 Forest plot of odds ratios of 30 day risk for stroke or death (individual end points) within 30 days of carotid endarterectomy versus carotid artery stenting. ICSS was not included in this analysis for death because overall mortality data based on an intention to treat analysis were not available See footnote to table for full title of studies

terminated prematurely. Five trials were stopped before they reached their predefined sample size (table). ${ }^{21-232629}$ These trials showed more pronounced superiority of carotid endarterectomy over carotid artery stenting (odds ratio $0.56,0.30$ to $1.0 ; \mathrm{P}=0.058$ ), whereas the difference was smaller in studies completed formally $(0.76,0.47$ to $1.23 ; \mathrm{P}=0.261)$.

\section{Secondary end points}

Periprocedural stroke was reported in nine studies, two of which did not observe any stroke during this period. The average weighted incidence of stroke was $4.2 \%$ (95\% confidence interval $2.7 \%$ to $6.1 \%$ ) for carotid endarterectomy versus $5.7 \%$ (3.0\% to $9.2 \%)$ for carotid artery stenting. The risk of stroke was significantly lower for carotid endarterectomy (odds ratio 0.65 , $95 \%$ confidence interval 0.43 to $1.00 ; \mathrm{P}=0.049$; $\mathrm{I}^{2}=48.4 \%, \mathrm{P}=0.071$ ) (fig 3).

Periprocedural mortality was reported as an end point in eight trials; in three, no death was observed in either group. ${ }^{8232527-3032}$ The average weighted incidence of death was $1.4 \%$ (95\% confidence interval $0.08 \%$ to $2.1 \%$ ) for carotid endarterectomy and $1.2 \%$ $(0.7 \%$ to $1.8 \%)$ for carotid artery stenting. Overall, there was no significant difference between patients treated with carotid endarterectomy versus carotid artery stenting (odds ratio 1.14, 95\% confidence interval 0.56 to $2.31 ; \mathrm{P}=0.727 ; \mathrm{I}^{2}=0 \% ; \mathrm{P}=0.697$ ) (fig 3).

Data on the composite end point of periprocedural disabling stroke or death was available from eight trials; no event was observed in two. ${ }^{8232527-3032}$ The weighted average event rate was $2.9 \%$ (95\% confidence interval $1.9 \%$ to $4.3 \%$ ) for carotid endarterectomy versus $3.8 \%$ (2.3\% to $5.7 \%)$ for carotid artery stenting. In the direct comparison, this difference was not significant (odds ratio $0.74,95 \%$ confidence interval 0.53 to $\left.1.05 ; \mathrm{P}=0.088 ; \mathrm{I}^{2}=0 \% ; \mathrm{P}=0.600\right)$. For the Stent-Supported Percutaneous Angioplasty of the Carotid Artery versus Endarterectomy (SPACE) trial, the composite end point was defined as ipsilateral disabling stroke or death; for the International Carotid Stenting Study (ICSS), only per protocol data were available for this end point and these data were used instead of intention to treat data.

The rate of periprocedural myocardial infarction was reported in four trials. ${ }^{25283233}$ The weighted average infarction rate was $2.6 \%$ (95\% confidence interval $0.4 \%$ to $6.3 \%$ ) for carotid endarterectomy versus $0.9 \%$ $(0.05 \%$ to $2.9 \%)$ for carotid artery stenting. The risk of infarction was significantly higher in the carotid endarterectomy group (odds ratio 2.69, 95\% confidence interval 1.06 to $6.79 ; \mathrm{P}=0.036 ; \mathrm{I}^{2}=0 \% ; \mathrm{P}=0.700$ ) (fig 4).

Six studies reported on periprocedural cranial facial neuropathy; no event occurred in one study. ${ }^{252728303233}$ The average weighted event rate was $7.5 \%$ (95\% confidence interval $5.8 \%$ to $9.4 \%$ ) for carotid endarterectomy versus $0.45 \%(0.01 \%$ to $1.0 \%)$ for carotid artery stenting. Overall, the risk was higher in the carotid endarterectomy group (odds ratio 10.25, $95 \%$ confidence interval 4.02 to 26.13 ; $\mathrm{P}<0.001$; $\mathrm{I}^{2}=0 \% ; \mathrm{P}=0.754$ ) (fig 5).

\section{Sensitivity analyses}

Analyses of periprocedural risk for major end points provided odds ratio with confidence intervals close to 1.0. Thus, reanalyses of the three end points death and stroke, death, and stroke were done by two additional meta-analytical approaches deemed meaningful in estimating variances (see web extra table 2). The point estimates for the odds ratio were similar and confidence intervals even narrower for most end points. The influence of individual studies was tested by reanalyses, omitting one study at a time. No study showed an overwhelming influence on the overall odds ratio estimates (data not presented).

Data from the ICSS trial also included myocardial infarction in the primary end point death or stroke; corresponding data without myocardial infarction were not available for the intention to treat analysis but were available as a per protocol analysis. Reanalysis using the per protocol data produced a similar result (odds ratio $0.63,95 \%$ confidence interval 0.42 to 0.94 ; $\left.\mathrm{P}=0.025 ; \mathrm{I}^{2}=47.2 \% ; \mathrm{P}=0.056\right)$.

We also carried out an analysis including trials that only enrolled patients with carotid stenosis who had symptoms while excluding the SAPPHIRE (Stenting and Angioplasty with Protection in Patients at High 


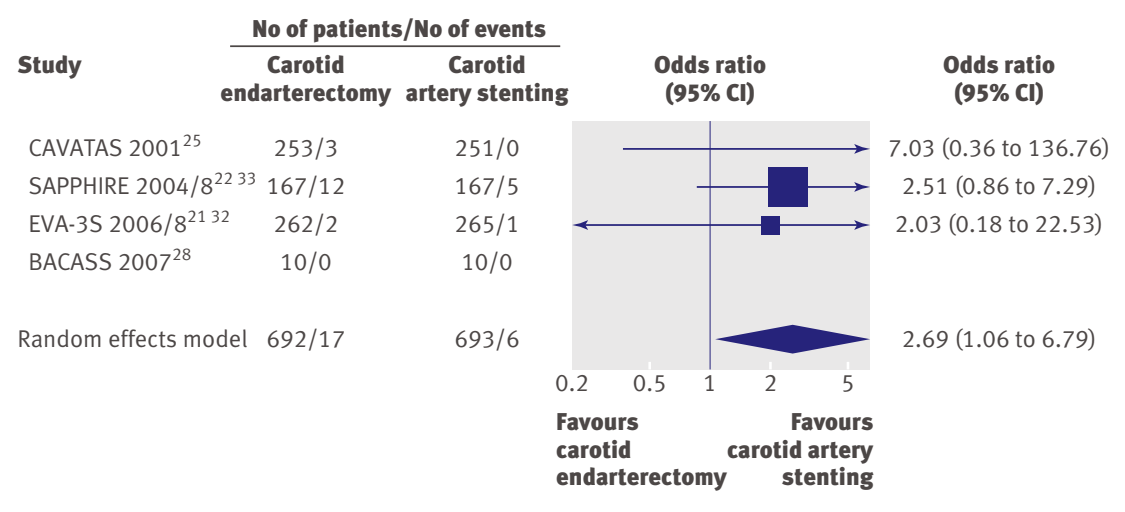

Fig 4 | Forest plot of odds ratios of 30 day risk for myocardial infarction for carotid endarterectomy versus carotid artery stenting. See footnote to table for full title of studies

Risk for Endarterectomy) trial ${ }^{2233}$ and Brooks et al's ${ }^{30}$ trial that predominantly or exclusively consisted of asymptomatic patients. For the primary end point the odds ratio for carotid endarterectomy versus carotid artery stenting was similar $(0.63,0.44$ to 0.92 ; $\mathrm{P}=0.017$ ).

\section{Intermediate term outcomes}

The risk for the intermediate term composite end point stroke or death did not differ significantly between carotid endarterectomy and carotid artery stenting. Among the nine trials reporting on long term results, hazard ratios were available for the four larger trials; data for the SAPPHIRE trial ${ }^{2233}$ were provided by the study investigators. For the data of the CAVATAS trial, ${ }^{25}$ the hazard ratios were controlled for sex, age, and trial centre, whereas the other studies provided unadjusted hazard ratios; furthermore, the hazard ratios of the CAVATAS trial were based on deaths and disabling strokes. Data on the ICSS trial ${ }^{8}$ were not considered because the maximum follow-up time was limited to 120 days. No difference was found between carotid artery stenting and carotid endarterectomy (hazard ratio $1.11,95 \%$ confidence interval 0.91 to $1.35 ; \mathrm{P}=0.315 ; \mathrm{I}^{2}=0 \% ; \mathrm{P}=0.400$ ) (fig 6).

We also analysed event data based on the classic incidence of events (as for short term data). Binary data (events and total number of patients per group) were available for nine trials and did not show any difference in outcome (odds ratio $0.87,95 \%$ confidence interval 0.71 to $1.07 ; \mathrm{P}=0.190 ; \mathrm{I}^{2}=0 \% ; \mathrm{P}=0.432$ ) (fig 7 ).

The risk of stroke in the intermediate term was not significantly different between the two treatments in the four studies providing information on time to event analyses (hazard ratio $0.86,95 \%$ confidence interval 0.67 to $1.10 ; \mathrm{P}=0.216 ; \mathrm{I}^{2}=4.3 \% ; \mathrm{P}=0.371$ ). The classic analysis based on eight trials providing binary data on this end point ${ }^{252728303233}$ gave an odds ratio of 0.78 (95\% confidence interval 0.56 to 1.09$) ; \mathrm{P}=0.151$ $\left(\mathrm{I}^{2}=12.3 \% ; \mathrm{P}=0.337\right)$. Intermediate term mortality did not differ significantly for carotid endarterectomy compared with carotid artery stenting for the trials (hazard ratio $1.09,0.76$ to $1.57 ; \mathrm{P}=0.625 ; \mathrm{I}^{2}=6.3 \%$; $\mathrm{P}=0.306)$, but time to event based data were available for only two trials. The classic analysis based on seven trials providing binary data on this end point $^{252728303233}$ gave an odds ratio of 1.04 (95\% confidence interval 0.78 to 1.38$) ; \mathrm{P}=0.779 \quad\left(\mathrm{I}^{2}=0 \%\right.$; $\mathrm{P}=0.856)$.

\section{DISCUSSION}

This meta-analysis indicates that carotid endarterectomy is associated with better periprocedural outcomes than carotid artery stenting. This disparity was primarily driven by a difference in risk of procedural non-disabling stroke, which seemed to be higher in patients treated with carotid artery stenting. Conversely, the risk of facial neuropathy and periprocedural myocardial infarction was higher with carotid endarterectomy. In the intermediate term to long term, primary or secondary end points did not differ significantly, suggesting similar efficacy of the two procedures.

Since carotid revascularisation is carried out solely for the prevention of future events, the rate of procedural complications (especially stroke) is a key driver of the risk-benefit ratio. The trade-off between the procedural risk and the longer term benefit has to be evaluated carefully; this also has to be kept in mind when interpreting trials comparing procedures for treating carotid artery stenosis; especially since none of the trials included a best medical therapy only group.

Carotid endarterectomy has been shown to reduce the overall risk for stroke and death compared with medical treatment in patients with relevant carotid artery stenosis both with symptoms ${ }^{34}$ and without symptoms ${ }^{35}$. Based on these trials, an acceptable upper limit of perioperative rate for stroke or death has been determined to be around 3\% for asymptomatic patients ${ }^{35}$ and $6 \%$ for patients with symptoms. ${ }^{3637}$ Congruent with this, our analysis of mainly patients with symptoms showed a procedure related event rate of $5.4 \%$ for carotid endarterectomy. For carotid artery stenting, the average perioperative event rate was $7.3 \%$ in our analysis. In this perspective, evaluation of expected excess benefit for an individual patient is difficult and has to be based on his or her estimated baseline risk of stroke without treatment and on life expectancy. Moreover, the efficiency of medical treatment has probably improved, since most of the pivotal trials on carotid endarterectomy did not prescribe to the aggressive risk factor reduction that would be considered standard today for patients with established atherosclerosis. ${ }^{38} 39$

Failure to account for trends in outcomes over time is a major limitation of a meta-analysis evaluating an emerging technology. This is evident in the specialty of cardiovascular medicine as the outcomes with carotid artery stenting have continued to improve and the results reported in recent registries have been superior to those in most of the trials included in the meta-analysis. Whereas the average 30 day rate for stroke or death was 7.3\% in the included trials, the Emboshield and Xact Post Approval Carotid Stent Trial (EX) registry (which included 2145 high risk surgical patients) and the Carotid ACCULINK/ACCUNET Post 


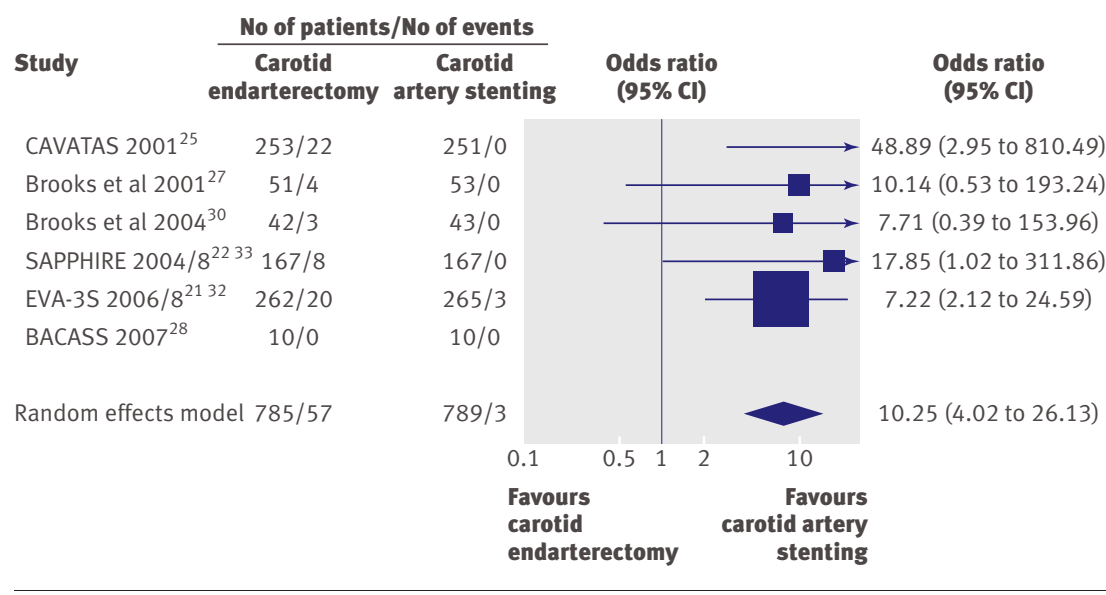

Fig 5 | Forest plot of odds ratio of periprocedural (30 day) cranial nerve injuries for carotid endarterectomy versus carotid artery stenting. See footnote to table for full title of studies

Approval Trial to Uncover Rare Events (C2) (which included 4175 patients) showed 30 day rates for stroke or death of only $4.1 \%$ and $3.4 \%$, respectively. ${ }^{40}$ This may be attributed to a learning curve in the emerging technique of carotid artery stenting with improvement in equipment design, optimisation of patient selection, adequate training of operators, and better attention to pharmacotherapy (see web extra fig 2). This learning curve has been a major drawback for some of the largest trials in carotid artery stenting where a significant proportion of operators had limited experience, 39\% of patients in the Endarterectomy Versus Angioplasty in patients with Symptomatic Severe carotid Stenosis (EVA-3S) trial were treated by doctors with modest experience of the procedure ${ }^{41}$ and use of emboli protection devices and dual antiplatelet therapy was not universal. ${ }^{2332}$

This would suggest that carotid artery stenting as carried out in most patients included in the meta-analysis would be expected to be inferior to carotid endarterectomy, whereas the intermediate term outcome would be similar. These findings have important implications for patients undergoing carotid revascularisation. Currently, use of carotid artery stenting in the United States is restricted by Centers for Medicare and Medicaid Services guidelines to patients at high risk for carotid endarterectomy or those enrolled in clinical trials. The results of this meta-analysis suggest that it may be premature to consider any revision to these guidelines. Furthermore, the consistently inferior short term results of carotid artery stenting as done in these studies suggests a need for a strategy aimed at minimising the risk of procedural stroke. This would involve adequate case selection, rigorous training and credentialing of operators, appropriate strategies to reduce procedural embolic phenomenon, and suitable drugs. Current trials must ensure that such mechanisms are in place and that patients are clearly informed of the risks in undergoing carotid artery stenting versus carotid endarterectomy. It is worth noting that factors rendering patients at high risk from carotid endarterectomy are not necessarily identical to those for carotid artery stenting. Although comorbidities are the major risk factors with carotid endarterectomy, carotid artery stenting outcomes are mainly influenced by the anatomy of local vessels, and both factors have to be considered in treatment decision making. ${ }^{42}$

Although there have been multiple recent meta-analysis in carotid revascularisation, ${ }^{643}$ our study significantly extends the work because of a larger population, a more robust methodology, and longer term follow-up data. Earlier studies have erroneously split composite end points from the SAPPHIRE trial, ${ }^{2233}$ which we confirmed from study authors. Also, previous meta-analysis preferentially reported on a fixed effect model instead of the more conservative random effect model, which is more appropriate here owing to considerable study heterogeneity. ${ }^{6}$ Furthermore, we used appropriate transformations to generate weighted proportions for procedural complication rates.

Finally, we used time to event analysis for longer term outcomes, and the pooled hazard ratio provides a more accurate assessment of events compared with odds ratios or risk ratios that ignore possible variation in time to events and incomplete follow-up. This is particularly relevant to events such as stroke or death where an event early in the study would be considerably more devastating than one occurring later.

\section{Heterogeneity of study results}

Even though heterogeneity of study results was moderate, relevant aspects of dissimilarities in study designs have to be considered. Most of the studies excluded asymptomatic patients whereas one study evaluated asymptomatic patients exclusively ${ }^{30}$ and in another trial two thirds of patients were asymptomatic. ${ }^{22}$ Moreover, average surgical risk of the study populations were different; the SAPPHIRE trial $^{22}$ included high risk patients exclusively. This trial, in contrast with most others, found a superiority of carotid artery stenting over carotid endarterectomy. This suggests that since intermediate term outcomes are similar it might be appropriate to select a revascularisation strategy based on the procedural risk of patients. However, exclusion of these two studies including predominantly asymptomatic patients did not change the overall outcome of our analysis.

A major reason for heterogeneity between the studies was because almost half of the trials were stopped prematurely, before the prespecified sample size was reached. Early stopping can lead to overestimation of treatment effects (stop at random high) as shown in several previous analyses. ${ }^{445}$ For two of the early stopped trials, however, the decision was not based on differential outcomes but on problems with funding ${ }^{23}$ or recruitment, ${ }^{22}$ whereas for three trials the early stopping was based on large differences in risk for the compared treatments. ${ }^{212629}$ These three trials may have led to some overestimation of the superiority of carotid endarterectomy. This fact highlights the importance of completion of currently ongoing trials - for example, CREST (Carotid Revascularization 


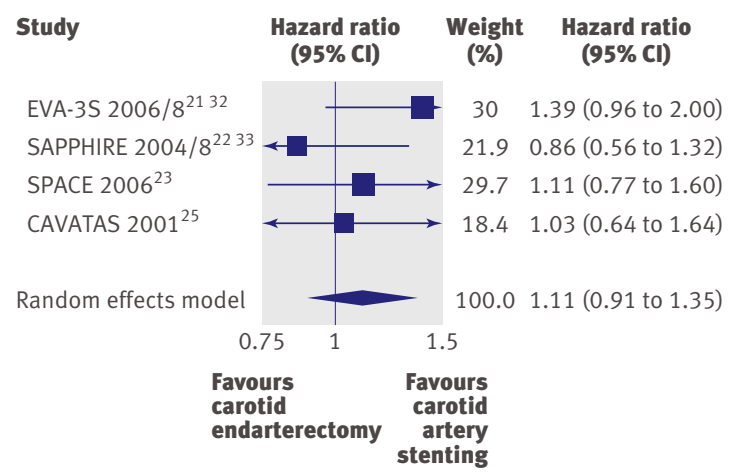

Fig 6 | Forest plot of hazard ratio of intermediate term risk for composite of stroke or death

Endarterectomy versus Stent Trial) and ACT 1 (Asymptomatic Carotid Stenosis, Stenting versus Endarterectomy Trial) - to define the best contemporary carotid revascularisation strategy.

Furthermore, concomitant drug treatment differed among the included trials. Earlier trials required aspirin treatment only ${ }^{29}$ with no pretreatment, whereas the Wallstent trial ${ }^{26}$ used ticlopidine for four weeks after the procedure, and more recent studies ${ }^{20232428}$ used clopidogrel for about 2-4 weeks after the procedure, often for 72 to 24 hours before the stenting procedure. This may be particularly relevant to the periprocedural outcomes for carotid stenting where inadequate platelet inhibition may predispose to early thrombosis and embolisation with the attendant hazard of stroke.

\section{Limitations of the meta-analysis}

Most of the patients in this meta-analysis had symptoms; asymptomatic patients were under-represented and a generalisation to this population would be speculative. Asymptomatic patients generally have a lower procedural risk, but specific evidence for this group is

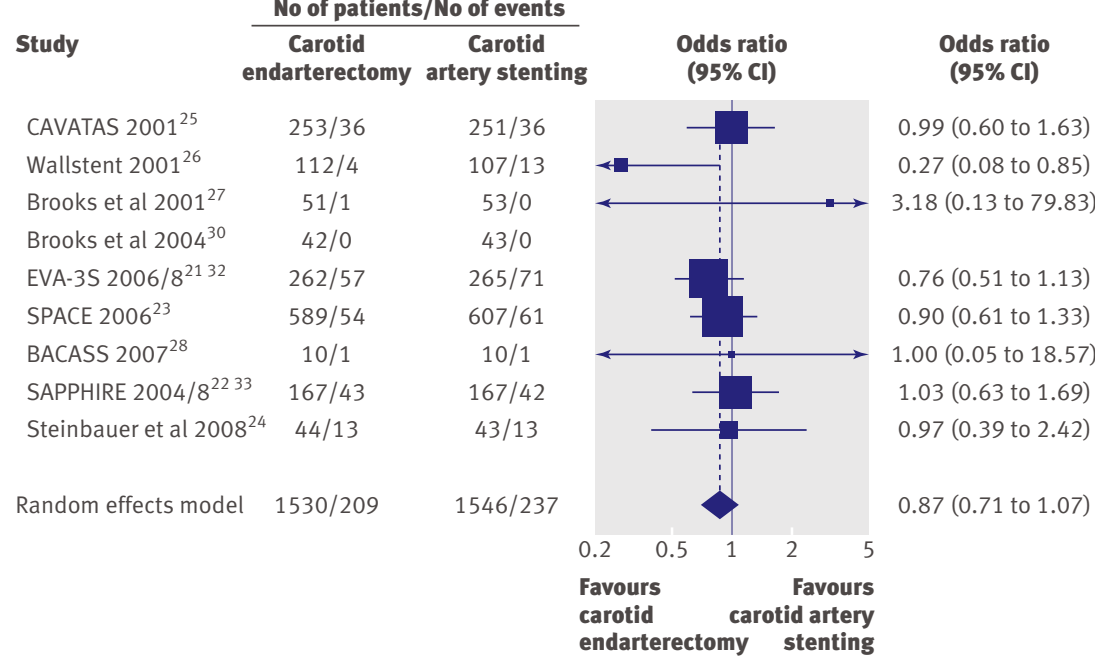

Fig 7 | Forest plot of odds ratios of intermediate term risk for composite of stroke or death as binary outcomes, without considering time interval between intervention and event (Wallstent trial $^{26}$ used an endpoint definition of "ipsilateral stroke, procedure-related death, or vascular death within 1 year") currently limited to one trial that did not observe an event in either group. ${ }^{30}$ Conceptually, preventive treatment of carotid artery disease rather than intervening after a first cerebrovascular event would seem meaningful, although a similar degree of prevention may be achieved by aggressive medical therapy. More data on this population are required, although contemporary registry studies suggest a persistently high risk of stroke in asymptomatic patients with carotid stenosis. ${ }^{46}$

Two of the included studies have only been presented at scientific meetings or published as abstracts and did not undergo a rigorous peer review process. The quality assessment of these studies is therefore limited. Exclusion of these trials from the analysis did not change the results. Finally, there are several limitations of the included studies, which may have introduced a systematic bias against carotid stenting and potentially led to an overestimation of the benefit of carotid endarterectomy. Firstly, the primary end point of most studies was a composite of death or stroke. This end point has been used traditionally for most trials in the discipline of carotid endarterectomy. This composite end point seems meaningful as an efficacy end point but neglects important safety issues such as periprocedural myocardial infarction and cranial neuropathy. The incidence of each of these events was less than $1 \%$ on average for carotid stenting in the included trials whereas for carotid endarterectomy the average incidence for myocardial infarction was $2.6 \%$ and that for cranial neuropathy was 7.5\%. Even though most of the cranial nerve neuropathies are transient, about $4 \%$ persist over several months and about $0.5 \%$ are permanent. ${ }^{47}$ Such injuries to the cranial nerve and also myocardial infarctions can have an effect on a patient's quality of life similar to that after a stroke. Future trials should consider the use of composite end points that also include relevant safety end points. Secondly, most included studies based their preprocedural assessment mainly on carotid duplex sonography, which generally gives an insufficient assessment of the vessel anatomy. Vascular anatomy is a key determinant of periprocedural risk for carotid artery stenting. However, the perioperative risk with carotid endarterectomy is mainly defined by clinical risk factors that are usually available at first encounter with patients and may have served to exclude high risk patients from inclusion in a study. This could potentially introduce a selection bias that disfavours carotid stenting. Finally, most trials required extensive experience of the surgeons carrying out carotid endarterectomy, whereas the corresponding requirements for interventionalists doing carotid stenting were less stringent. The EVA-3S trials, for example, required surgeons to have carried out at least 25 procedures in the previous year, whereas interventionalists had to have carried out at least 12 carotid stenting procedures overall or only five if they had done 35 stentings of the supra-aortic trunk; those who had less experience could enrol patients with a tutor, who was required to have carried out at least 12 procedures overall. Thus 


\section{WHAT IS ALREADY KNOWN ON THIS TOPIC}

Several randomised controlled trials have compared procedural outcomes of carotid artery stenting with carotid endarterectomy

The results of such trials have been controversial

Little is known about intermediate term outcomes of carotid stenting and carotid endarterectomy

\section{WHAT THIS STUDY ADDS}

In this meta-analysis carotid endarterectomy was found to be superior to coronary artery stenting for short term outcomes

The difference was not significant for intermediate term outcomes

Significantly fewer cranial nerve injuries and myocardial infarctions occurred with carotid stenting compared with carotid endarterectomy

the lack of similar operator experience may have biased the results against carotid stenting.

\section{Conclusion}

In our meta-analysis including 4796 patients with carotid stenosis predominantly with symptoms, carotid endarterectomy was associated with a lower risk for the primary end point of death or stroke. This difference was mainly driven by the lower incidence of periprocedural non-disabling or minor strokes. A relevant temporal impact exists indicating a continuously improving outcome for the newer carotid artery stenting approach compared with the longer established carotid endarterectomy. We found no significant differences in numbers of major or disabling strokes or deaths, whereas significantly fewer cranial nerve injuries and myocardial infarctions occurred with carotid artery stenting. Intermediate term outcomes were not significantly different between the two interventions. Patients with symptoms requiring carotid revascularisation should currently be offered carotid endarterectomy as first choice, with carotid artery stenting reserved for patients at high surgical risk. Trials of contemporary carotid artery stenting versus carotid endarterectomy are needed to better understand the role for each treatment in patients with or without symptoms.

We thank Whitney Townsend (librarian, Taubman Medical Library, University of Michigan) for her inputs and help during the literature search and Don Cutlip (Beth Israel Hospital, Harvard Medical School) for providing data on the SAPPHIRE trial.

Contributors: HSG and PM conceived and designed the study. HSG, PM, GK, and SC analysed and interpreted the data. PM drafted the paper. HSG, SC, GK, and UT revised the paper critically for important intellectual content. All authors had full access to the data (including statistical reports and tables) in the study and take responsibility for the integrity of the data and the accuracy of the data analysis. All authors also approved the final version to be published. HSG and PM are guarantors for this study

Funding: PM is supported by a postdoctoral fellowship grant from the Swiss National Research Foundation and the Schweizerische Stiftung fü Medizinisch-Biologische Stipendien. The funding organisation had no role in the design and conduct of the study; the collection, management, analysis, and interpretation of the data; or the preparation, review, or approval of the manuscript.

Competing interests: SC is a consultant for Abbott Vascular.

Ethical approval: Not required.

Data sharing: Datasets and statistical code for R and SAS are available from the corresponding author at hgurm@umich.med.edu.
1 Linfante I, Andreone V, Akkawi N, Wakhloo AK. Internal carotid arten stenting in patients over 80 years of age: single-center experience and review of the literature. / Neuroimaging 2009;19:158-63.

2 Van der Vaart MG, Meerwaldt R, Reijnen MM, Tio RA, Zeebregts CJ. Endarterectomy or carotid artery stenting: the quest continues. $\mathrm{Am}$ ) Surg 2008;195:259-69.

3 Chambers BR, Donnan GA. Carotid endarterectomy for asymptomatic carotid stenosis. Cochrane Database Syst Rev 2005;(4):CD001923.

4 Yadav JS, Roubin GS, lyer S, Vitek J, King P, Jordan WD, et al. Elective stenting of the extracranial carotid arteries. Circulation 1997;95:376-81.

5 Bonaldi G. Angioplasty and stenting of the cervical carotid bifurcation: report of a 4-year series. Neuroradiology 2002;44:164-74.

6 Ederle J, Featherstone RL, Brown MM. Randomized controlled trials comparing endarterectomy and endovascular treatment for carotid artery stenosis: a Cochrane systematic review. Stroke 2009;40:1373-80

7 Sidawy AN, Zwolak RM, White RA, Siami FS, Schermerhorn ML, Sicard GA. Risk-adjusted 30-day outcomes of carotid stenting and endarterectomy: results from the SVS Vascular Registry. I Vasc Surg 2009;49:71-9.

8 European Stroke Conference. Safety results of the International Carotid Stenting Study (ICSS): early outcome of patients randomised between carotid stenting and endarterectomy for symptomatic carotid stenosis. Sweden, 2009. www.ion.ucl.ac.uk/cavatas_icss/ downloads/FirstResultsoflCSS.pdf.

9 Meier P, Hayward RA. Regarding "Risk-adjusted 30-day outcomes of carotid stenting and endarterectomy: results from the SVS Vascular Registry." I Vasc Surg 2009;50:475.

10 Moher D, Liberati A, Tetzlaff J, Altman DG. Preferred reporting items for systematic reviews and meta-analyses: the PRISMA statement. PLoS Med 2009;6:e1000097.

11 Juni $\mathrm{P}$, Witschi A, Bloch R, Egger $M$. The hazards of scoring the quality of clinical trials for meta-analysis. JAMA 1999;282:1054-60.

12 Knapp G, Hartung J. Improved tests for a random effects metaregression with a single covariate. Stat Med 2003;22:2693-710.

13 Hartung J, Knapp G, Sinha BK. Statistical meta-analysis with applications. Wiley-Interscience, 2008.

14 Sutton AJ, Higgins JP. Recent developments in meta-analysis. Stat Med 2008;27:625-50.

15 Sankey S, Weissfeld L, Fine M, Kapoor W. An assessment of the use of the continuity correction for sparse data in metanalysis. Commun Stat: Simul Comput 1996;25:1031-56.

16 Begg C, Mazumdar M. Operating characteristics of a rank correlation test for publication bias. Biometrics 1994;50:1088-101.

17 Egger M, Davey Smith G, Schneider M, Minder C. Bias in metaanalysis detected by a simple, graphical test. BMJ 1997;315:629-34.

18 Rucker G, Schwarzer G, Carpenter J. Arcsine test for publication bias in meta-analyses with binary outcomes. Stat Med 2008;27:746-63.

19 Miller JJ. The inverse of the Freeman-Tukey double arcsine transformation. Am Stat 1978;32:138.

20 R Development Core Team. R: a language and environment for statistical computing. R Foundation for Statistical Computing, Vienna, Austria, 2009. www.R-project.org.

21 Mas IL, Trinquart L, Leys D, Albucher JF, Rousseau H, Viguier A, et al. Endarterectomy versus angioplasty in patients with symptomatic severe carotid stenosis (EVA-3S) trial: results up to 4 years from a randomised, multicentre trial. Lancet Neurol 2008;7:885-92.

22 Gurm HS, Yadav JS, Fayad P, Katzen BT, Mishkel GJ, Bajwa TK, et al. Long-term results of carotid stenting versus endarterectomy in highrisk patients. N Engl J Med 2008;358:1572-9.

23 Ringleb PA, Allenberg J, Bruckmann H, Eckstein HH, Fraedrich G, Hartmann M, et al. 30 day results from the SPACE trial of stentprotected angioplasty versus carotid endarterectomy in symptomatic patients: a randomised non-inferiority trial. Lancet 2006;368:1239-47.

24 Steinbauer MG, Pfister K, Greindl M, Schlachetzki F, Borisch I, Schuirer G, et al. Alert for increased long-term follow-up after carotid artery stenting: results of a prospective, randomized, single-center trial of carotid artery stenting vs carotid endarterectomy. J Vasc Surg 2008;48:93-8.

25 Endovascular versus surgical treatment in patients with carotid stenosis in the Carotid and Vertebral Artery Transluminal Angioplast Study (CAVATAS): a randomised trial. Lancet 2001;357:1729-37.

26 Alberts M. Results of a multicenter prospective randomized trial of carotid artery stenting vs carotid endarterectomy. Stroke 2001;32:325-d

27 Brooks WH, McClure RR, Jones MR, Coleman TC, Breathitt L. Carotid angioplasty and stenting versus carotid endarterectomy: randomized trial in a community hospital. J Am Coll Cardio 2001;38:1589-95.

28 Hoffmann A, Engelter S, Taschner C, Mendelowitsch A, Merlo A, Radue EW, et al. Carotid artery stenting versus carotid endarterectomy-a prospective randomised controlled single-centre 
trial with long-term follow-up (BACASS). Schweiz Arch Neurol Psychiatr 2008;159:84-9.

29 Naylor AR, Bolia A, Abbott RJ, Pye IF, Smith J, Lennard N, et al. Randomized study of carotid angioplasty and stenting versus carotid endarterectomy: a stopped trial. J Vasc Surg 1998;28:326-34.

30 Brooks WH, McClure RR, Jones MR, Coleman TL, Breathitt L. Carotid angioplasty and stenting versus carotid endarterectomy for treatment of asymptomatic carotid stenosis: a randomized trial in a community hospital. Neurosurgery 2004;54:318-24.

31 CaRESS Steering Committee. Carotid revascularization using endarterectomy or stenting systems (CaRESS) phase I clinical trial: 1 year results. J Vasc Surg 2005;42:213-9.

32 Mas JL, Chatellier G, Beyssen B, Branchereau A, Moulin T, Becquemin JP, et al. Endarterectomy versus stenting in patients with symptomatic severe carotid stenosis. N Engl J Med 2006;355:1660-71.

33 Yadav JS, Wholey MH, Kuntz RE, Fayad P, Katzen BT, Mishkel GJ, et al. Protected carotid-artery stenting versus endarterectomy in high-risk patients. N Engl J Med 2004;351:1493-501.

34 Cina CS, Clase CM, Haynes RB. Carotid endarterectomy for symptomatic carotid stenosis. Cochrane Database Syst Rev 2000;(2):CD001081.

35 Chambers BR, You RX, Donnan GA. Carotid endarterectomy for asymptomatic carotid stenosis. Cochrane Database Syst Rev 2000;(2):CD001923.

36 Randomised trial of endarterectomy for recently symptomatic carotid stenosis: final results of the MRC European Carotid Surgery Trial (ECST). Lancet 1998;351:1379-87.

37 Barnett HJ, Taylor DW, Eliasziw M, Fox AJ, Ferguson GG, Haynes RB, et al. Benefit of carotid endarterectomy in patients with symptomatic moderate or severe stenosis. N Engl J Med 1998;339:1415-25.
38 Sillesen H, Amarenco P, Hennerici MG, Callahan A, Goldstein LB, Zivin J, et al. Atorvastatin reduces the risk of cardiovascular events in patients with carotid atherosclerosis: a secondary analysis of the stroke prevention by aggressive reduction in cholesterol levels (SPARCL) trial. Stroke 2008;39:3297-302.

39 Chaturvedi S. Should the multicenter carotid endarterectomy trials be repeated? Arch Neurol 2003;60:774-5.

40 Gray WA, Chaturvedi S, Verta P. Thirty-day outcomes for carotid artery stenting in 6320 patients from 2 prospective, multicenter, highsurgical-risk registries. Circ Cardiovasc Interv 2009:159-66.

41 Maree AO, Rosenfield KA. Endarterectomy versus stenting for carotid stenosis. N Engl J Med 2007;356:306.

42 Bates ER, Babb JD, Casey DE Jr, Cates CU, Duckwiler GR, Feldman TE, et al. ACCF/SCAI/SVMB/SIR/ASITN 2007 clinical expert consensus document on carotid stenting. Vasc Med 2007;12:35-83.

43 Gurm HS, Nallamothu BK, Yadav J. Safety of carotid artery stenting for symptomatic carotid artery disease: a meta-analysis. Eur Heart J 2008;29:113-9.

44 Meier P, Ko DT, Tamura A, Tamhane U, Gurm HS. Sodium bicarbonate-based hydration prevents contrast-induced nephropathy: a meta-analysis. BMC Med 2009;7:23.

45 Montori VM, Devereaux PJ, Adhikari NK, Burns KE, Eggert CH, Briel M, et al. Randomized trials stopped early for benefit: a systematic review. JAMA 2005;294:2203-9.

46 Aichner FT, Topakian R, Alberts MJ, Bhatt DL, Haring HP, Hill MD, et al. High cardiovascular event rates in patients with asymptomatic carotid stenosis: the REACH registry. Eur J Neurol 2009;16:902-8.

47 Cunningham El, Bond R, Mayberg MR, Warlow CP, Rothwell PM. Risk of persistent cranial nerve injury after carotid endarterectomy. J Neurosurg 2004;101:445-8.

Accepted: 14 December 2009 